\title{
The role of pion exchange in $\eta$ meson photoproduction on the deuteron
}

\author{
A. $\operatorname{Fix}^{1 *}$, H. Arenhövel ${ }^{2 \dagger}$, M. Levchuk ${ }^{3 \ddagger}$, and M. Tammam ${ }^{4 \S}$ \\ ${ }^{1}$ Laboratory of Mathematical Physics, Tomsk Polytechnic University, 634034 Tomsk, Russia \\ ${ }^{2}$ Institut für Kernphysik, Johannes Gutenberg-Universität Mainz, D-55099 Mainz, Germany \\ ${ }^{3}$ Stepanov Institute of Physics, Belarus National Academy of Sciences, 220072 Minsk, Belarus and \\ ${ }^{4}$ Physics Department, Al-Azhar University, Assiut, Egypt
}

(Dated: November 7, 2018)

\begin{abstract}
Incoherent $\eta$ photoproduction on the deuteron is studied with the main emphasis on the role of final state interactions. In addition to the previously studied mechanisms of $N N$ and $\eta N$ rescatterings, the role of an intermediate pion exchange is considered in detail, where first a pion is photoproduced on one nucleon and then rescatters into an $\eta$ meson on the other, the spectator nucleon. It is found, that the role of this pion mediated contribution is comparable in size to that of $\eta N$ rescattering. Results for total and semi-inclusive differential cross sections and associated polarization observables are presented. In particular polarization observables show a significant sensititvity to final state interactions.
\end{abstract}

PACS numbers: 25.20.Lj, 13.60.Le, 14.20.Gk

\section{INTRODUCTION}

Photoproduction of an $\eta$ meson on a deuteron was investigated quite extensively in Refs. [1 [9]. In general, the focus was on the question of how much the influence of final state interactions (FSI) overshadows the information on the single nucleon response by studying $\eta$ photoproduction on quasifree nucleons, in particular, on a neutron. It has been shown that the interaction effects are rather important in the near-threshold region but become less significant with increasing photon energy in the unpolarized total cross section. Thus at higher energies the impulse approximation (IA, diagram (a) of Fig. 1), associated with the one-nucleon response, provides the dominant part of the total cross section. However, for the differential cross section the FSI mechanisms which involve at least two nucleons are expected to be of importance in the region of large momentum transfer, primarily at backward $\eta$ emission angles. Indeed, in this region the energy $\Delta E$ transferred by the produced meson is essentially lower than the energy transfer associated with quasifree kinematics, that is $\Delta E \ll(\Delta p)^{2} / 2 M_{N}$, where $\Delta p$ is the corresponding momentum transfer and $M_{N}$ the nucleon mass. This imbalance results in a suppression of the single nucleon response in the region of high $\Delta p$ values. In this situation mechanisms in which two or more nucleons can share the transferred momentum become increasingly important. As the dominant reaction mode in this kinematic region one can expect the rescattering of the final two nucleons (diagram (b) of Fig. 1). The role of the $\eta N$ rescattering (diagram (c) of Fig. 1) was already considered in Refs. [2, 4, 9] and was shown to be quite important, too.

If in addition pion degrees of freedom are considered, then $\eta$ photoproduction can proceed according to the two-step scheme $\gamma N \rightarrow \pi N \rightarrow \eta N$ (diagram (d) of Fig. 1), where the photoproduced pion is subsequently rescattered into an $\eta$ meson by the spectator nucleon. To the best of our knowledge, there is no thorough investigation of this mechanism for this reaction. In general, it was simply ignored, since it was argued that the corresponding amplitude should be suppressed due to a large momentum of the intermediate pion. Namely, because the pion has a relatively small mass, its propagation is associated with a large intermediate momentum and, therefore, should be effective only at short internucleon distances. For example, the $\pi N N$ channel provides only a small fraction of the $\eta d$ scattering cross section, which is governed by the long-range structure of the $\eta N N$ wave function (see Ref. [5]).

Although these arguments appear reasonable, the insignificance of pion rescattering in incoherent $\eta$ photoproduction is not obvious since, as already noted above, at higher energies are kinematic regions where the two-nucleon response becomes essential. Furthermore, the cross section for $\gamma N \rightarrow \pi N$ is much larger than that for $\gamma N \rightarrow \eta N$ so that the above noted suppression due to a large intermediate momentum can be compensated by a high yield of pion photoproduction. It is therefore important to study the role of this pion exchange via a direct inclusion of the corresponding diagram (d) of Fig. 1 into the reaction amplitude.

\footnotetext{
* Electronic address: fix@tpu.ru

$\dagger$ Electronic address: arenhoev@kph.uni-mainz.de

‡ Electronic address: levchuk@dragon.bas-net.by

$\S$ Electronic address: mmatm@yahoo.com
} 


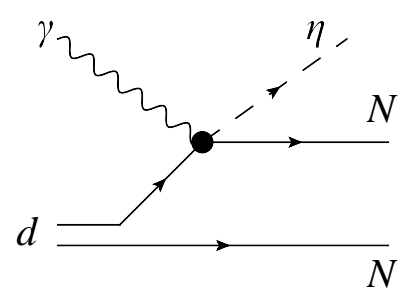

(a)

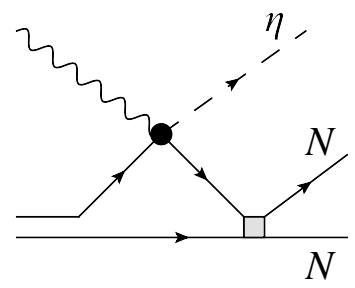

(b)

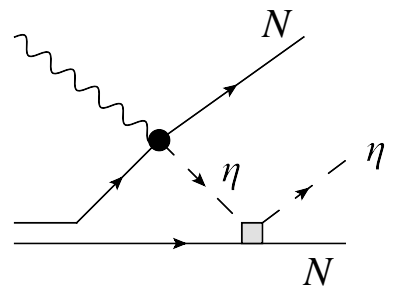

(c)

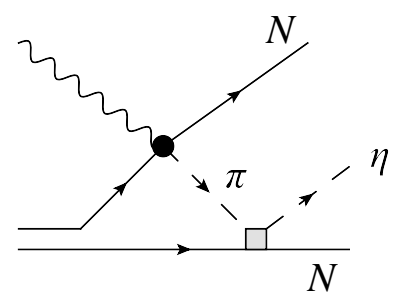

(d)

FIG. 1: Diagrammatical representation of the amplitude for $\gamma d \rightarrow \eta n p$ : (a) Impulse (spectator) approximation (IA), (b) $N N$ rescattering, (c) $\eta N$ rescattering, and (d) pion exchange mechanism $(\pi N)$.

Furthermore, the influence of such a pion exchange in kaon photoproduction on a deuteron was investigated rather detailed in Ref. [10], where it has been shown that in some charge channels its inclusion can visibly change even the total cross section. Namely, in $K^{+}$photoproduction the pion mediated process $\gamma d \rightarrow \pi N N \rightarrow K \Sigma N$ results in an almost 10-15\% increase of the total cross section in the maximum and thus seems to be the most important FSI mechanism in this reaction.

One should note that in some of the previous studies the contribution of such pion exchange was already partially investigated. However, the corresponding results suffer from some quite rough approximations. For example, in Ref. [8], where a three-body $\eta N N-\pi N N$ approach was used, only the resonance $S_{11}(1535)$ in the pion photoproduction operator was included. This can lead to a significant underestimation of the role of the pion exchange, since even in the region of the $S_{11}(1535)$ this resonance plays a very moderate role in pion photoproduction. Furthermore, in an earlier paper [2], a simple approximation with respect to the choice of energy and momentum of the initial nucleon in the deuteron was adopted (see the formalism in Ref. 2]). Such a treatment was partially justified since the $\pi$ exchange as well as $\eta$ rescattering are indeed rather insignificant in the total cross section, to which this paper was devoted, so that already such a rough estimation of their role was sufficient to draw some conclusions about the reaction dynamics. Therefore, in view of these crude approximations for the pion exchange mechanism in previous studies, it appears timely to bring the theory to an appropriate quantitative level.

In the next section we briefly describe the most important dynamical ingredients of the reaction matrix, in particular, those aspects concerning the pion rescattering mechanism. Sect. III reviews briefly the general form of the observables in terms of the $T$-matrix. In Sect. [V] we present our results for the unpolarized total cross section and associated polarization asymmetries, the semi-inclusive differential cross section and some selected corresponding polarization observables, which are especially sensitive to FSI effects. Some conclusions and an outlook are given in Sect. VV.

\section{THE T-MATRIX}

We consider the following reaction

$$
\gamma\left(E_{\gamma}, \vec{k}\right)+d\left(E_{d}, \vec{p}_{d}\right) \rightarrow \eta\left(\omega_{\eta}, \vec{q}_{\eta}\right)+N\left(E_{1}, \vec{p}_{1}\right)+N\left(E_{2}, \vec{p}_{2}\right),
$$

where the four-momenta of the participating particles are given in parenthesis. As reference frame we choose the overall center-of-mass (c.m.) system with $z$-axis along the incoming photon momentum and the $x$-axis along the direction of maximal linear photon polarization. Total and differential cross sections and associated polarization observables as well are determined by the $T$-Matrix element

$$
T_{s m_{s} t \lambda m_{d}}=-{ }^{(-)}\left\langle\vec{p}_{1} \vec{p}_{2} s m_{s}, t, \vec{q}\left|\vec{\varepsilon}_{\lambda} \cdot \vec{J}_{\gamma \eta}(0)\right| \vec{p}_{d} 1 m_{d}\right\rangle
$$

between the deuteron ground state with spin projection $m_{d}$ on the $z$-axis and the final two-nucleon state with total spin $s=0,1$, its projection $m_{s}$ and total isospin $t=0,1$. The index $\lambda= \pm 1$ denotes the circular photon polarization.

The calculation of the matrix element (2) follows the standard recipe in which the spectator model (IA) is treated as the first basic approximation, and the interaction between the final particles is taken into account in the form of additional terms which are presented by the diagrams (b) through (d) in Fig. 1 Thus the resulting amplitude is the sum of the four contributions

$$
T=T^{I A}+T^{N N}+T^{\eta N}+T^{\pi N},
$$


where the last term corresponds to the pion exchange mechanism which is the main object of the present investigation. The procedure, which we used to calculate the first three terms, is described in a number of papers [3, 4, 7, 9], and we refer the reader to these for more details.

The elementary $\eta$ photoproduction operator $t_{\gamma \eta}$, entering the first three terms in Eq. (3) is taken from the isobar model EtaMAID [11]. The latter includes contributions from Born terms, vector meson exchanges in the $t$-channel, and $s$-channel resonances $D_{13}(1520), S_{11}(1535), S_{11}(1650), D_{15}(1675), F_{15}(1680), D_{13}(1700), P_{11}(1710)$, and $P_{13}(1720)$. This model provides a reasonable description of the available data on $\eta$ photo- and electroproduction on the nucleon in the energy region up to a total c.m. energy $W=2 \mathrm{GeV}$, which corresponds to a lab photon energy $E_{\gamma}^{\text {lab }}=$ $1650 \mathrm{MeV}$. In the energy region of the present study from threshold up to $E_{\gamma}^{l a b}=800 \mathrm{MeV}$, the resonance $S_{11}(1535)$ dominates the cross section, and the other resonances are of minor importance only. The off-mass shell behavior of the photoproduction operator was taken according to the energy-momentum conservation at the single-nucleon vertex

$$
\begin{aligned}
\vec{p}_{i n} & =\vec{q}_{\eta}+\vec{p}_{1}+\vec{p}_{2}-\vec{k}, \\
E_{i n} & =\omega_{\eta}+E_{1}+E_{2}-E_{\gamma},
\end{aligned}
$$

where $\left(E_{i n}, \vec{p}_{i n}\right)$ denotes the four-momentum of the initial nucleon in the deuteron. In all cases, the $\eta$ meson and both nucleons were taken on-shell.

The amplitude $T^{\pi N}$ in (3) has the form

$$
T^{\pi N}=\left\langle s, m_{s}, t\left|t_{\pi \eta}\left(N_{2}\right) G_{\pi N N} t_{\gamma \pi}^{\lambda}\left(N_{1}\right)\right| 1 m_{d}\right\rangle-(-1)^{t+s}(1 \leftrightarrow 2),
$$

where $t_{\gamma \pi}^{\lambda}\left(N_{i}\right)$ and $t_{\pi \eta}\left(N_{i}\right), i=1,2$ denote the amplitudes for photoproduction of a pion and for $\pi N_{i} \rightarrow \eta N_{i}$ rescattering on a nucleon $N_{i}$, respectively. $G_{\pi N N}$ is the propagator of the three free particles $\pi, N_{1}$, and $N_{2}$. For $t_{\gamma \pi}^{\lambda}$ we took the MAID2007 amplitudes [12]. With respect to its isospin structure one has

$$
t_{\gamma \pi}^{\lambda}=A^{(0)}-2 A^{(-)}, \quad \text { for } t=0
$$

and

$$
t_{\gamma \pi}^{\lambda}=A^{(+)}+2 A^{(0)}, \quad \text { for } t=1
$$

with $t$ denoting the total isospin of the two nucleons. The amplitudes $A^{(0, \pm)}$ are those which appear in the isospin decomposition of the pion photoproduction amplitude according to

$$
t_{\gamma \pi}^{\lambda}(N)=A^{(+)} \delta_{b 3}+\frac{1}{2} A^{(-)}\left[\tau_{b}, \tau_{3}\right]+A^{(0)} \tau_{b},
$$

with $b$ being the isotopic index of the produced pion.

In order to calculate $\eta N \rightarrow \eta N$ and $\pi N \rightarrow \eta N$ scatterings we use an isobar ansatz from Ref. [9], which is driven exclusively by the dominant $S_{11}(1535)$ resonance, parametrized in the form

$$
t_{\alpha \beta}\left(\omega_{\eta N}, \vec{q}, \vec{q}^{\prime}\right)=\frac{g_{\alpha}(\vec{q}) g_{\beta}\left(\vec{q}^{\prime}\right)}{\omega_{\eta N}-M_{0}-\Sigma_{\eta}\left(\omega_{\eta N}\right)-\Sigma_{\pi}\left(\omega_{\eta N}\right)+\frac{i}{2} \Gamma_{\pi \pi}}, \quad \alpha, \beta \in\{\pi, \eta\},
$$

where the $S_{11}(1535)$ self energies, $\Sigma_{\eta}$ and $\Sigma_{\pi}$, are given by

$$
\Sigma_{\alpha}\left(\omega_{\eta N}\right)=\frac{1}{2 \pi^{2}} \int \frac{q^{2} d q}{2 \omega_{\alpha}(q)} \frac{g_{\alpha}^{2}(q)}{\omega_{\eta N}-E_{N}(q)-\omega_{\alpha}(q)+i \epsilon}, \quad \alpha \in\{\pi, \eta\} .
$$

Here, $\omega_{\eta N}$ denotes the invariant $\eta N$ energy, and $E_{N}(q)=\sqrt{q^{2}+M_{N}^{2}}$ and $\omega_{\alpha}(q)=\sqrt{q^{2}+M_{\alpha}^{2}}$ the on-shell energies of nucleon and meson $\alpha$, respectively. The vertex functions $g_{\alpha}(q)$ are taken in a Hulthén form

$$
g_{\alpha}(q)=\frac{g_{\alpha}}{1+\left(q / \beta_{\alpha}\right)^{2}}
$$

with $\beta_{\alpha}$ as a cut-off momentum. The two-pion channel is included in a simplified manner by adding the $S_{11} \rightarrow \pi \pi N$ decay width in the form

$$
\Gamma_{\pi \pi}=\gamma_{\pi \pi} \frac{W-M_{N}-2 m_{\pi}}{m_{\pi}}
$$



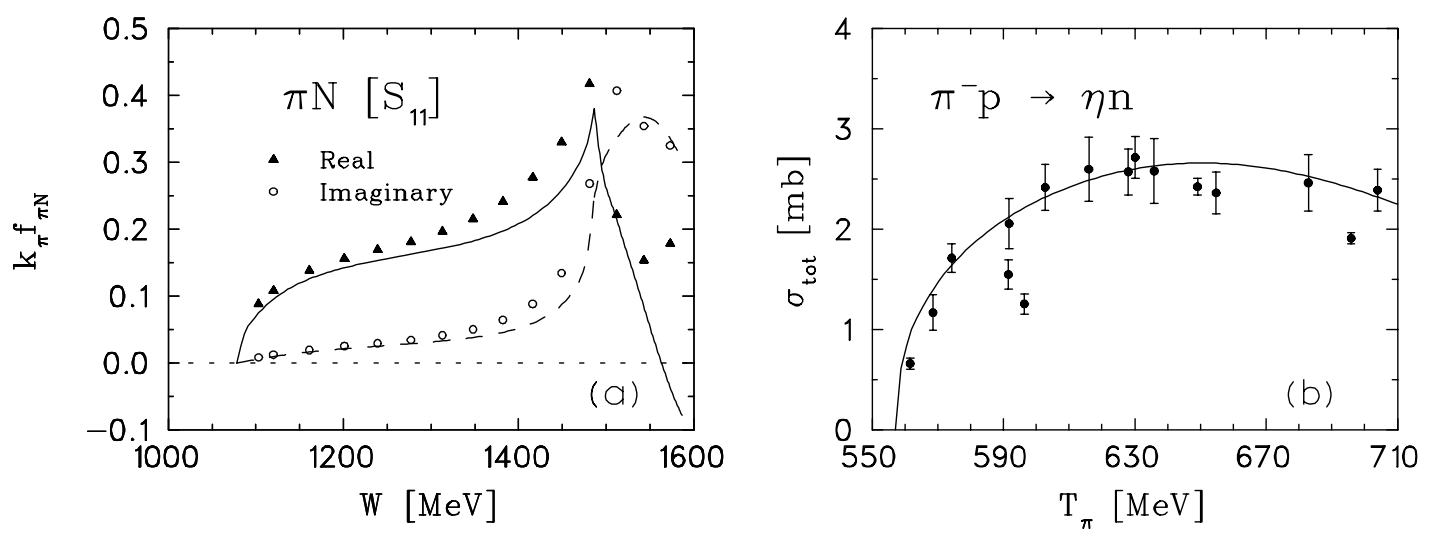

FIG. 2: Left panel (a): $S_{11}$ partial wave amplitude $\mathrm{f}_{\pi N}$ for $\pi N$ scattering predicted by our parametrization of the $S_{11}(1535)$ resonance [see Eqs. (9) through (11)] as function of the total c.m. energy $W$. The amplitude is multiplied with the pion momentum $\mathrm{k}_{\pi}$. Notation: Solid curve : real part, dashed: imaginary part. Circles and triangles represent the VPI analysis [16]. Right panel (b): Total $\pi^{-} p \rightarrow \eta n$ cross section. The data are taken from the compilation in Ref. 13].

with $\gamma_{\pi \pi}=4.3 \mathrm{MeV}$, and $M_{N}$ and $m_{\pi}$ denoting nucleon and pion masses, respectively. The parameters $M_{0}, g_{\alpha}$, and $\beta_{\alpha}$ were adjusted in such a way that the $\eta N$ scattering length

$$
a_{\eta N}=(0.5+i 0.32) \mathrm{fm}
$$

is reproduced. This may be considered on one side as an approximate average of the scattering lengths given by modern $\eta N$ scattering analyses [13 15], and on the other side providing a reasonably good description of the reactions in the channels coupled to the $\eta N$ system as is shown in Fig. 2] The fit parameters are listed in Table 1 of Ref. [9].

In order to evaluate the $N N$ scattering amplitude as well as the deuteron wave function we have used the separable representation of the Paris potential according to Ref. 17]. All partial waves up to $J=2$ were taken into account. Furthermore, in order to check the dependence of the results on the choice of the $N N$ scattering model we carried out the same calculations with the CD-Bonn model [18] as well as with two versions of the Nijmegen potential (Nijm93 and NijmII) from Ref. [19] and found the results to be very close to those obtained with the separable Paris potential of Ref. [17]. The deviation is less than $10 \%$ in the near threshold region and decreases rapidly with increasing energy. This confirms the results of Refs. [3, 4, 7, 9] that the calculation is practically insensitive to the choice of the $N N$ potential.

\section{OBSERVABLES}

The general formalism for cross section and polarization observables for photoproduction of a pseudoscalar meson on a deuteron is presented in detail in Ref. 20]. From this work we take the general expressions for total and semiinclusive differential cross sections in the c.m. system, when only the direction of the produced $\eta$ meson, characterized by $\Omega_{\eta}=\left(\theta_{\eta}, \phi_{\eta}\right)$, is measured,

$$
\begin{aligned}
\frac{d^{2} \sigma}{d \Omega_{\eta}}= & \frac{d^{2} \sigma_{0}}{d \Omega_{\eta}}\left[1+P_{1}^{d} T_{11}^{0} \sin \phi_{\eta d} d_{10}^{1}\left(\theta_{d}\right)+P_{2}^{d} \sum_{M=0}^{2} T_{2 M}^{0} \cos M \phi_{\eta d} d_{M 0}^{2}\left(\theta_{d}\right)\right. \\
& +P_{\ell}^{\gamma}\left\{\Sigma^{\ell} \cos 2 \phi_{\eta}+P_{1}^{d} \sum_{M=-1}^{1} T_{1 M}^{\ell} \sin \psi_{M} d_{M 0}^{1}\left(\theta_{d}\right)+P_{2}^{d} \sum_{M=-2}^{2} T_{2 M}^{\ell} \cos \psi_{M} d_{M 0}^{2}\left(\theta_{d}\right)\right\} \\
& \left.+P_{c}^{\gamma}\left\{P_{1}^{d} \sum_{M=0}^{1} T_{1 M}^{c} \cos M \phi_{\eta d} d_{M 0}^{1}\left(\theta_{d}\right)+P_{2}^{d} \sum_{M=1}^{2} T_{2 M}^{c} \sin M \phi_{\eta d} d_{M 0}^{2}\left(\theta_{d}\right)\right\}\right]
\end{aligned}
$$

Here $P_{\ell}^{\gamma}$ and $P_{c}^{\gamma}$ denote the degrees of linear and circular photon polarization, and $P_{1}^{d}$ and $P_{2}^{d}$ the deuteron vector and tensor polarization of the deuteron target with respect to an axis characterized by $\Omega_{d}=\left(\theta_{d}, \phi_{d}\right)$. Furthermore, we 
have abbreviated for convenience $\phi_{\eta d}=\phi_{\eta}-\phi_{d}$ and $\psi_{M}=M \phi_{\eta d}-2 \phi_{\eta}$. The unpolarized semi-inclusive differential cross section and the associated asymmetries are given by

$$
\begin{aligned}
\frac{d^{2} \sigma_{0}}{d \Omega_{\eta}} & =V_{00}^{1}\left(\theta_{\eta}\right), \\
\Sigma^{\ell} \ell\left(\theta_{\eta}\right) \frac{d^{2} \sigma_{0}}{d \Omega_{\eta}} & =W_{00}\left(\theta_{\eta}\right), \\
T_{11}^{0}\left(\theta_{\eta}\right) \frac{d^{2} \sigma_{0}}{d \Omega_{\eta}} & =-2 \operatorname{Im}\left[V_{11}^{1}\left(\theta_{\eta}\right)\right], \\
T_{I M}^{c}\left(\theta_{\eta}\right) \frac{d^{2} \sigma_{0}}{d \Omega_{\eta}} & =-\left(2-\delta_{M 0}\right) \operatorname{Im}\left[i^{-\delta_{I 1}} V_{I M}^{1}\left(\theta_{\eta}\right)\right], \quad \text { for } 0 \leq M \leq I, \\
T_{I M}^{\ell}\left(\theta_{\eta}\right) \frac{d^{2} \sigma_{0}}{d \Omega_{\eta}} & =i^{\delta_{I 1}} W_{I M}\left(\theta_{\eta}\right), \quad \text { for }-I \leq M \leq I .
\end{aligned}
$$

The quantities $V_{I M}^{1}$ and $W_{I M}$ are hermitean quadratic forms in the $T$-matrix elements integrated over the $\eta$ momentum $q_{\eta}$ and the angle $\Omega_{p}$ of the relative momentum $\vec{p}=\left(p, \Omega_{p}\right)$ of the final two-nucleons according to

$$
\begin{aligned}
W_{I M}\left(\theta_{\eta}\right)= & -\frac{\hat{I}}{\sqrt{3}} \int d q_{\eta} d \Omega_{p} c_{k i n} \sum_{m_{d} m_{d}^{\prime}}(-)^{1-m_{d}}\left(\begin{array}{ccc}
1 & 1 & I \\
m_{d}^{\prime} & -m_{d} & M
\end{array}\right) \\
& \sum_{s m_{s} t} t_{s m_{s} t 1 m_{d}^{\prime}}^{*}\left(q_{\eta}, \theta_{\eta}, \theta_{p}, \phi_{p \eta}\right) t_{s m_{s} t-1 m_{d}}\left(q_{\eta}, \theta_{\eta}, \theta_{p}, \phi_{p \eta}\right), \\
V_{I M}^{1}\left(\theta_{\eta}\right)= & \frac{\hat{I}}{\sqrt{3}} \int d q_{\eta} d \Omega_{p} c_{k i n} \sum_{m_{d} m_{d}^{\prime}}(-)^{1-m_{d}}\left(\begin{array}{ccc}
1 & 1 & I \\
m_{d}^{\prime} & -m_{d} & M
\end{array}\right) \\
& \sum_{s m_{s} t} t_{s m_{s} t 1 m_{d}^{\prime}}^{*}\left(q_{\eta}, \theta_{\eta}, \theta_{p}, \phi_{p \eta}\right) t_{s m_{s} t 1 m_{d}}\left(q_{\eta}, \theta_{\eta}, \theta_{p}, \phi_{p \eta}\right),
\end{aligned}
$$

with $\phi_{p \eta}=\phi_{p}-\phi_{\eta}$. Here the small $t$-matrix elements are defined by

$$
t_{s m_{s} t \lambda m_{d}}=e^{-i\left(\lambda+m_{d}-m_{s}\right) \phi_{\eta}} T_{s m_{s} t \lambda m_{d}},
$$

and $c_{k i n}$ denotes a kinematic factor

$$
c_{k i n}=\frac{1}{(2 \pi)^{5}} \frac{E_{d} M_{N}^{2} p q_{\eta}^{2}}{4 E_{\gamma} W \omega_{N N} \omega_{\eta}}
$$

where the total energy in the $\gamma d$ c.m. system and the invariant energy of the final two-nucleon subsystem are denoted by $W$ and $\omega_{N N}$, respectively.

The total cross section with inclusion of photon and target polarization effects is obtained by integrating $d^{2} \sigma / d \Omega_{\eta}$ over the meson spherical angle $\Omega_{\eta}$ and reads

$$
\begin{aligned}
\sigma\left(P_{\ell}^{\gamma}, P_{c}^{\gamma}, P_{1}^{d}, P_{2}^{d}\right)= & \sigma_{0}\left[1+P_{2}^{d} \bar{T}_{20}^{0} \frac{1}{2}\left(3 \cos ^{2} \theta_{d}-1\right)+P_{c}^{\gamma} P_{1}^{d} \bar{T}_{10}^{c} \cos \theta_{d}\right. \\
& \left.+P_{\ell}^{\gamma} P_{2}^{d} \bar{T}_{22}^{\ell} \cos \left(2 \phi_{d}\right) \frac{\sqrt{6}}{4} \sin ^{2} \theta_{d}\right],
\end{aligned}
$$

where the unpolarized total cross section and the corresponding asymmetries are given by

$$
\begin{aligned}
\sigma_{0} & =\int d \Omega_{\eta} \frac{d^{2} \sigma_{0}}{d \Omega_{\eta}}, \\
\sigma_{0} \bar{T}_{I M}^{\alpha} & =\int d \Omega_{\eta} \frac{d^{2} \sigma_{0}}{d \Omega_{\eta}} T_{I M}^{\alpha},
\end{aligned}
$$

with $\alpha \in\{0, \ell, c\}$. 


\section{RESULTS AND DISCUSSION}

\section{A. Total cross section}

We start the discussion with the total unpolarized cross section and the associated asymmetries for polarized beam and target. The theoretical results for the different approximations together with available experimental data are presented in the left panel of Fig. 3 whereas the right panel shows the ratios with respect to the complete calculation.

In the near-threshold region the interaction effects are very important as one can clearly see in the panel (b). The resulting enhancement is about a factor 5 at the photon energy $640 \mathrm{MeV}$. As was earlier noted in Refs. [4, 9], the reason of such a strong influence of FSI is twofold. Firstly, the interaction between the final particles allows to balance the strong mismatch between the momentum needed for $\eta$ production on a nucleon in the deuteron and the characteristic internal nucleon momentum within the deuteron. We recall that in the impulse approximation the $\eta$ meson is produced only through the high momentum components of the target wave function, which appears in the deuteron with a small probability. Thus, this momentum mismatch is balanced mainly by the two-body $N N$ and to a lesser extent by the $\eta N$ FSI, producing a very large enhancement of the $\eta$ production rate. The second reason is an appreciable attraction in the $N N$ as well as in the $\eta N$ system which leads to an additional enhancement of the resulting cross section.
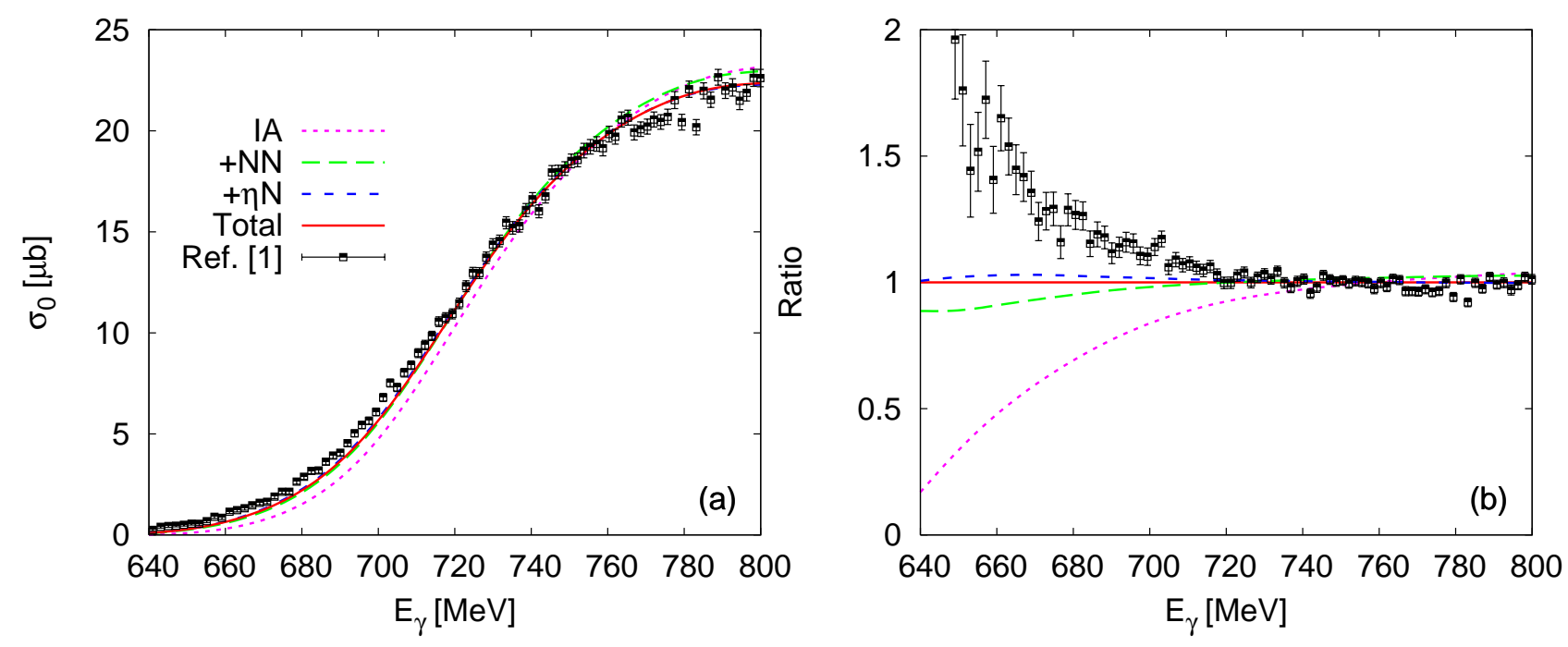

FIG. 3: (Color online) Left panel (a): Unpolarized total cross section $\sigma_{0}$ for $\gamma d \rightarrow \eta n p$. The dotted (magenta), long-dashed (green) and short-dashed (blue) curves correspond to the impulse approximation (IA) and successive inclusion of $N N$ and $\eta N$ rescatterings, respectively. The solid curve (Total, red) includes also the pion exchange mechanism. Experimental data from Ref. [1]. Right panel (b): Ratios of the various approximations with respect to the "Total" one.

As for the pion exchange mechanism (diagram (d) of Fig. 1), it plays at lower energies only a minor role. Its inclusion leads to a decrease of the cross section of about $3 \%$ at $E_{\gamma}^{l a b}=650 \mathrm{MeV}$. As already noted in the Introduction, this rather modest role of the pion exchange is expected as a consequence of the large characteristic momentum of the intermediate pion. Namely, if we assume that the largest contributions come from states where the pion is approximately on-shell, than its momentum is about $400 \mathrm{MeV} / \mathrm{c}$. The corresponding mechanism is effective only at small internucleon distances, about $0.5 \mathrm{fm}$, which are not essential for the incoherent channel. At the same time, as will be discussed in the next subsection, these mechanisms should be rather effective in the region of backward $\eta$ emission angles $\theta_{\eta}$ where large momentum transfers naturally emphasize small internucleon distances.

As one readily notes in the right panel of Fig. 3. even after inclusion of all FSI effects our calculation systematically underestimates the data in the region $E_{\gamma}^{l a b}<720 \mathrm{MeV}$. This question was discussed in quite some detail in Ref. [9]. In this work the reaction $\gamma d \rightarrow \eta n p$ was calculated within a three-body model, and it has been shown that the perturbative approach, in which $\eta N N$ interaction is reduced to pairwise $N N$ and $\eta N$ rescatterings, is unable to provide an accurate description of the reaction dynamics at low energy. The main reason for this fact is a relatively strong attraction which generates virtual poles in the $s$-wave $\eta N N$ states $J^{\pi}=1^{-}, T=0$ and $J^{\pi}=0^{-}, T=1[5]$. Obviously, the corresponding singularities in the $\eta N N$ scattering amplitude cannot be generated by a truncated 

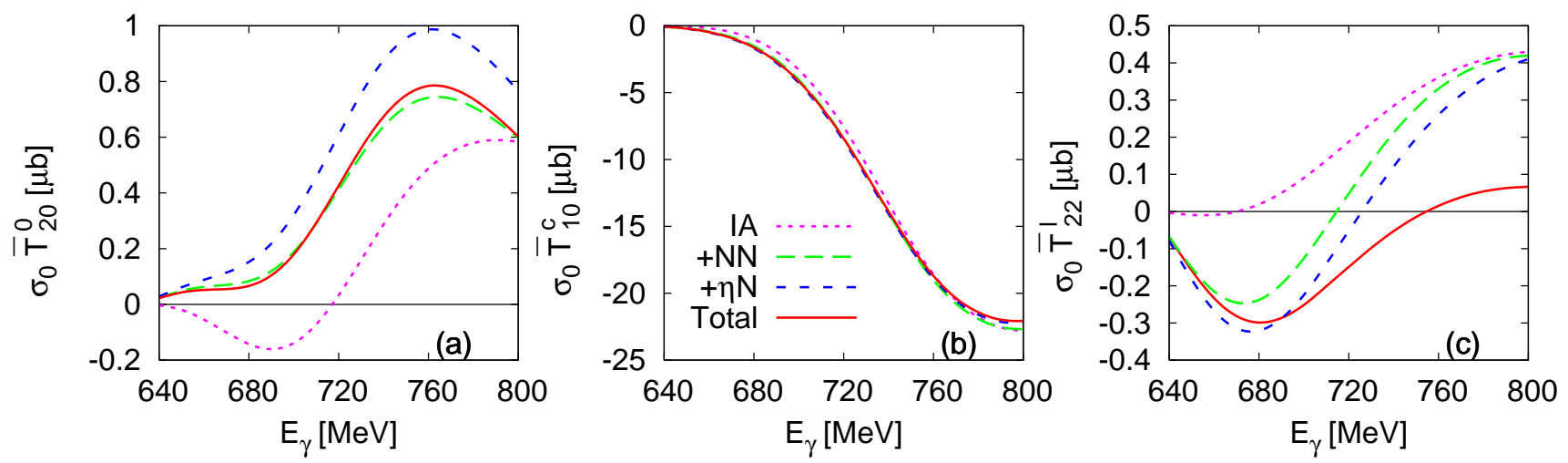

FIG. 4: (Color online) Polarization asymmetries of the total cross section for $\gamma d \rightarrow \eta n p$ : (a) $\sigma_{0} \bar{T}_{20}^{0}$, (b) $\sigma_{0} \bar{T}_{10}^{c}$, and (c) $\sigma_{0} \bar{T}_{22}^{\ell}$. Notation as in Fig. 3 .

perturbation expansion. According to the results of Refs. [6, [9], the inclusion of "three-body" effects leads to a visible improvement of the theory and brings the calculation into a better agreement with the data. Nevertheless, even in this case the theoretical total cross section still underestimates the data of Ref. [1] slightly.

At higher energies the effect of pion exchange becomes comparable in size to that coming from $\eta N$ rescattering. Despite the already mentioned rather large characteristic momentum of the intermediate pion, which reduces its contribution near threshold, the relatively large value of the pion photoproduction cross section at these higher energies appears to compensate this effect and makes the pion exchange more important although still quite small in the unpolarized total cross section.

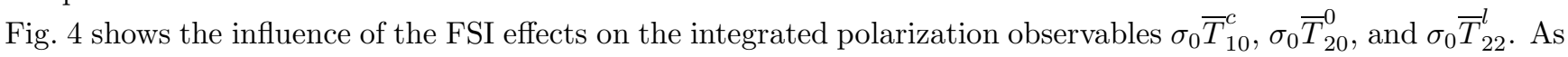
mentioned above, these are the only polarization observables contributing to the total cross section. The largest one is

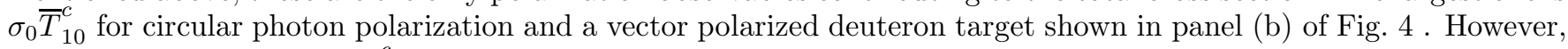
the influence of FSI on $\sigma_{0} \bar{T}_{10}^{c}$ is rather weak and comparable in size to that noted for the total cross sections. This observable determines the contribution of eta photoproduction to the Gerasimov-Drell-Hearn sum rule (GDH) [21].

For the other two observables in panels (a) and (c) of Fig. 4 the FSI influence is much more notable. For $\sigma_{0} \bar{T}_{20}^{0}$ (panel (a) of Fig. (4) for unpolarized photons and a tensor polarized target the contributions from $\eta N$ rescattering and pion exchange appear to compensate each other, leaving $N N$ rescattering as the main effect. Finally, $\sigma_{0} \bar{T}_{22}^{\ell}$ needing linearly polarized photons and again a tensor polarized target, $N N$ rescattering is dominant at low energies but dies out rapidly with increasing energy whereas the pion exchange contribution shows just the opposite behavior becoming dominant at higher energies. The contribution from $\eta N$ rescattering remains moderate in the whole energy range. Thus, these latter two observables would provide an interesting test for the study of the various FSI effects.

\section{B. Semi-inclusive differential cross section}

As next we will consider the semi-inclusive differential cross section of Eq. (14), for which only the direction of the produced $\eta$ meson is detected. In Fig. 5 the angular distributions predicted by our calculation are plotted for three energies together with the experimental data of Krusche et al. 1]. As expected the FSI effects are most notable in the region of backward angles, where the momentum transfer is maximal and, as a result, the two-nucleon response becomes important. To show the relative size of the different FSI mechanisms we plot in the lower panels of Fig. 5 the ratios of the various approximations with respect to the complete calculation, labelled "Total". One readily notes that at backward angles $\theta_{\eta}$ the inclusion of FSI effects leads to a sizable increase over the IA cross section. For energies $E_{\gamma} \geq 720 \mathrm{MeV}$ and at backward angles, i.e. in the region $\cos \theta_{\eta} \leq-0.5$, the effect of pion exchange becomes comparable in size to $\eta N$ rescatterings, but acting in the opposite direction. On the other hand, the angular distributions clearly show that the $\eta$ meson is produced mostly at forward direction, where the influence of all FSI mechanisms remains small and thus the IA works quite well (except in the region below $720 \mathrm{MeV}$ ).

In Figs. [6] and 7 we present for two energies a few polarization observables of the differential cross section in Eq. (14), which are in general more sensitive to dynamical details of the reaction. We have chosen those asymmetries which 

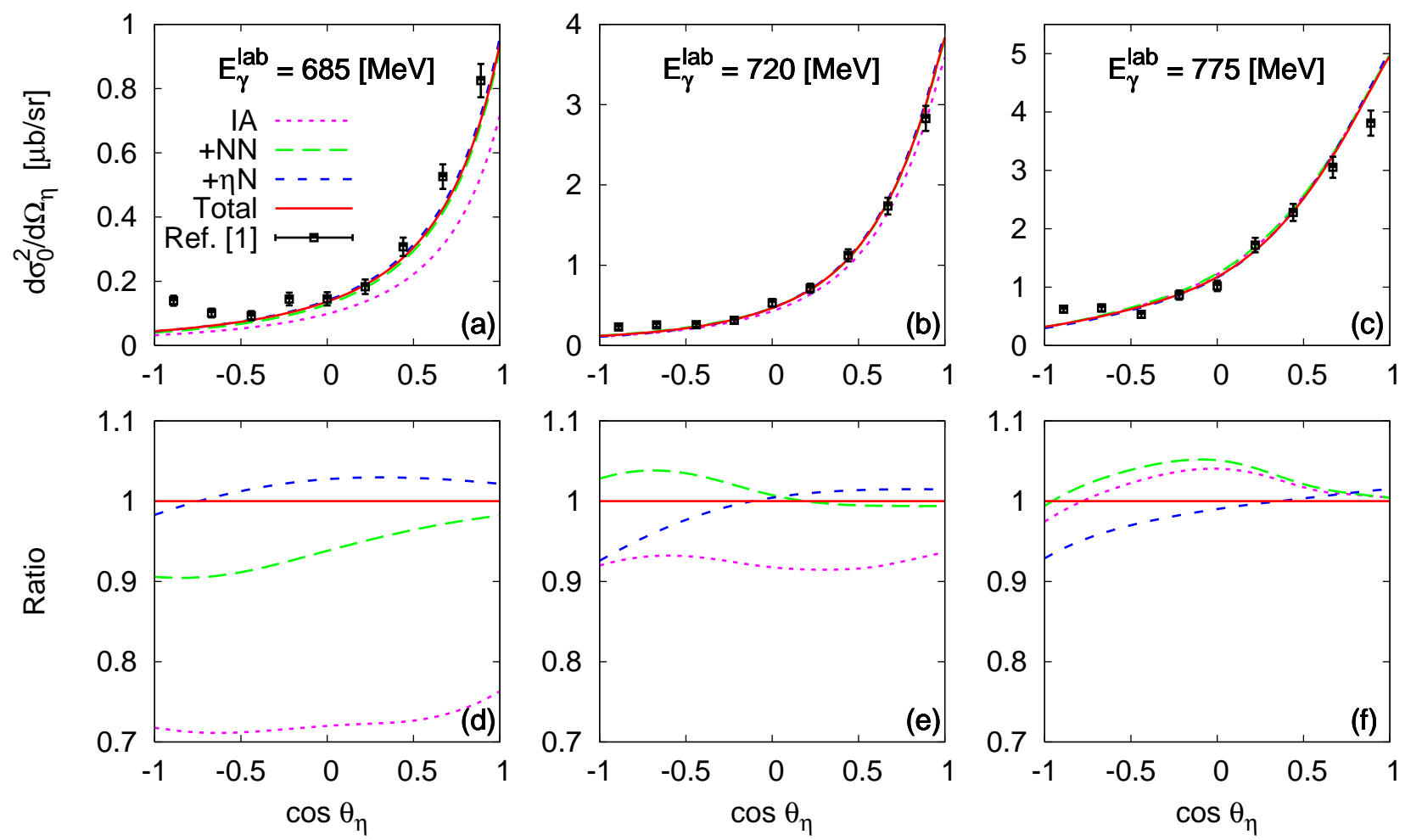

FIG. 5: (Color online) Semi-inclusive unpolarized differential cross section for $\gamma d \rightarrow \eta n p$ at the energies $E_{\gamma}^{l a b}=685 \mathrm{MeV}$ (a), $720 \mathrm{MeV}$ (b) and $775 \mathrm{MeV}$ (c). The data are from Ref. 1]. The lower panels show the corresponding ratios of the cross sections with respect to the complete calculation "Total". Notation as in Fig. 3

have relatively large values in the energy region, considered.

Fig. 6 exhibit $\Sigma$, the asymmetry for linearly polarized photons and an unpolarized target, $T_{11}^{0}$ for unpolarized radiation and a vector polarized deuteron target, and $T_{10}^{c}$, the asymmetry for circular photon polarization and again a vector polarized target. The latter asymmetry determines the GDH sum rule, mentioned above. The linear photon asymmetry $\Sigma$ is very little affected by FSI already at the lower energy of $685 \mathrm{MeV}$ (panel (a)) and even less at $775 \mathrm{MeV}$ (panel (d)). The other two observables in Fig. 6 are more sensitive to FSI, at least for the lower energy. For $T_{11}^{0}$ the influence of $N N$ rescattering at $685 \mathrm{MeV}$ (panel (b)) is quite small in contrast to a significant decrease of the IA by $\eta N$ rescattering. This decrease is more than counterbalanced by the pion exchange having the largest contribution and which leads to a significant overall increase compared to the IA. At the higher energy this sensitivity is lost (panel (e)). Turning to $T_{10}^{c}$ in panel (c) one readily notes that for this lower energy $N N$ rescattering is dominant at small angles, where the other effects are negligible, leading to an overall negative increase. At larger angles $\eta N$ rescattering becomes the dominant effect yielding a further sizeable negative increase at $180^{\circ}$. Pion exchange is small and acts opposite to $\eta N$ rescattering. At the higher energy all interaction effects become considerably smaller (see panel (f)). Pion exchange becomes comparable to $\eta N$ rescattering but almost cancels the latter, so that the overall FSI influence is very small.

The polarization observables shown in Fig. 7 for a tensor polarized deuteron target exhibit a stronger sensitivity to interaction effects, in particular for the lower energy (panels (a) through (c)). For the two observables $T_{20}^{0}$ and $T_{22}^{\ell}$ in panels (a) and (b) $N N$ rescattering is again dominant at forward angles with all other contributions negligible. However, at backward angles both $\eta N$ rescattering and pion exchange become quite sizeable, but cancelling each other partially. This cancellation is complete for $T_{21}^{c}$ in panel (c), leaving as net effect $N N$ rescattering. At the higher energy of $775 \mathrm{MeV}$ the overall influence of FSI diminishes substantially for $T_{20}^{0}$ in panel (d), while they remain stronger for $T_{22}^{\ell}$ and $T_{21}^{c}$ (panels (e) and (f)). In particular $\eta N$ rescattering appears quite strong for backward angles in both observables. But also pion exchange is sizeable.

In general, these polarization observables exhibit quite an interesting sensitivity to interaction effects, in many cases quite stronger than for the unpolarized angular distribution and thus offer an interesting possibility to study 

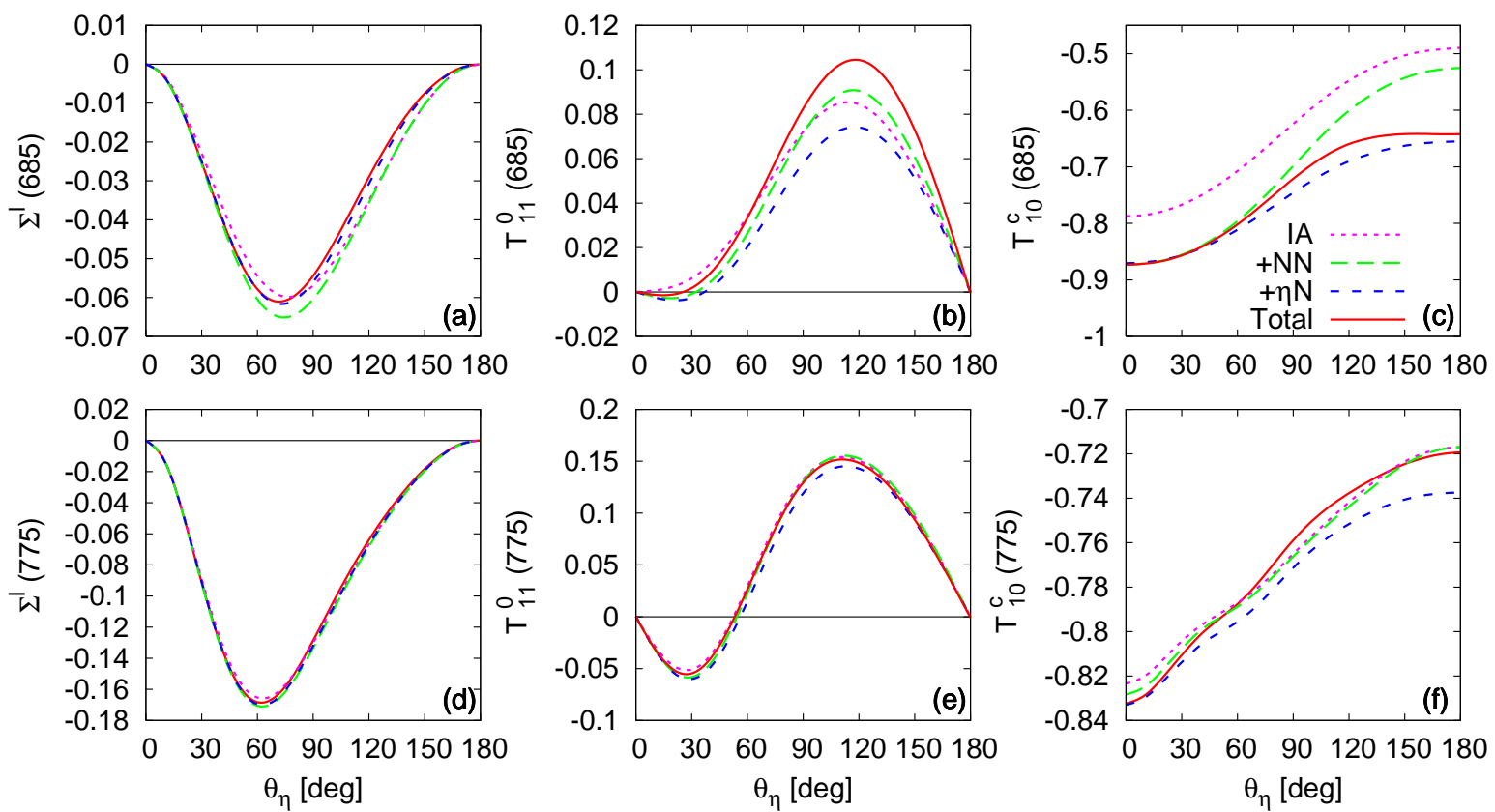

FIG. 6: (Color online) Polarization observables $\Sigma^{\ell}, T_{11}^{0}$, and $T_{10}^{c}$ of the semi-inclusive differential cross section for $\gamma d \rightarrow \eta n p$ at $E_{\gamma}^{l a b}=685$ (panels (a) through (c)) and $775 \mathrm{MeV}$ (panels (d) through (f)). Notation as in Fig. 3

this reaction in much greater detail.

\section{CONCLUSION}

In the present work we have considered incoherent photoproduction of $\eta$ mesons on the deuteron in the energy region from threshold up to $E_{\gamma}^{l a b}=800 \mathrm{MeV}$, with inclusion of the most important FSI mechanisms, $N N$ and $\eta N$ rescattering, as well as the pion exchange contribution $(\pi N)$. Our main purpose was to determine the role of the pion exchange without resorting to the approximations used in previous work. In general, as has also been shown by previous studies, the role of FSI in this reaction is rather unimportant for the total unpolarized cross section, except in the region just above threshold $\left(E_{\gamma}^{l a b}<720 \mathrm{MeV}\right)$. But this conclusion is not valid for the associated tensor target asymmetries, where FSI effects become significant, i.e. for $\bar{T}_{20}^{0}$ and $\bar{T}_{22}^{\ell}$, the latter for linearly polarized photons.

Furthermore, sizeable FSI effects become significantly visible also at higher energies in the differential cross section in those kinematical regions, where the spectator-nucleon mechanism is suppressed, for example for backward eta emission angles in the semi-inclusive differential cross section.

We also considered the role of FSI effects on different polarization observables. As our calculation shows, some of these observables, in particular $T_{10}^{c}, T_{20}^{0}$, and $T_{22}^{l}$ appear to be relatively large in the energy region considered in this work and, at the same time, are rather sensitive to FSI effects.

As a general conclusion, we would like to stress the fact that in the region $E_{\gamma}^{l a b} \geq 720 \mathrm{MeV}$ the pion exchange mechanism appears to be of the same size as $N N$ and $\eta N$ rescattering and should be taken into account using a reliable model. Furthermore, it remains to be seen in the futur whether the inclusion of pion degrees of freedom within a more realistic three-body approach than in Ref. [8], improves the agreement between theory and experiment in the near-threshold region.

\section{Acknowledgment}

The work was supported by the Deutsche Forschungsgemeinschaft (SFB 1044). A. Fix also acknowledges support from the Dynasty foundation, the TPU grant LRU-FTI-123-2014 and the MSU program 'Nauka' (project 

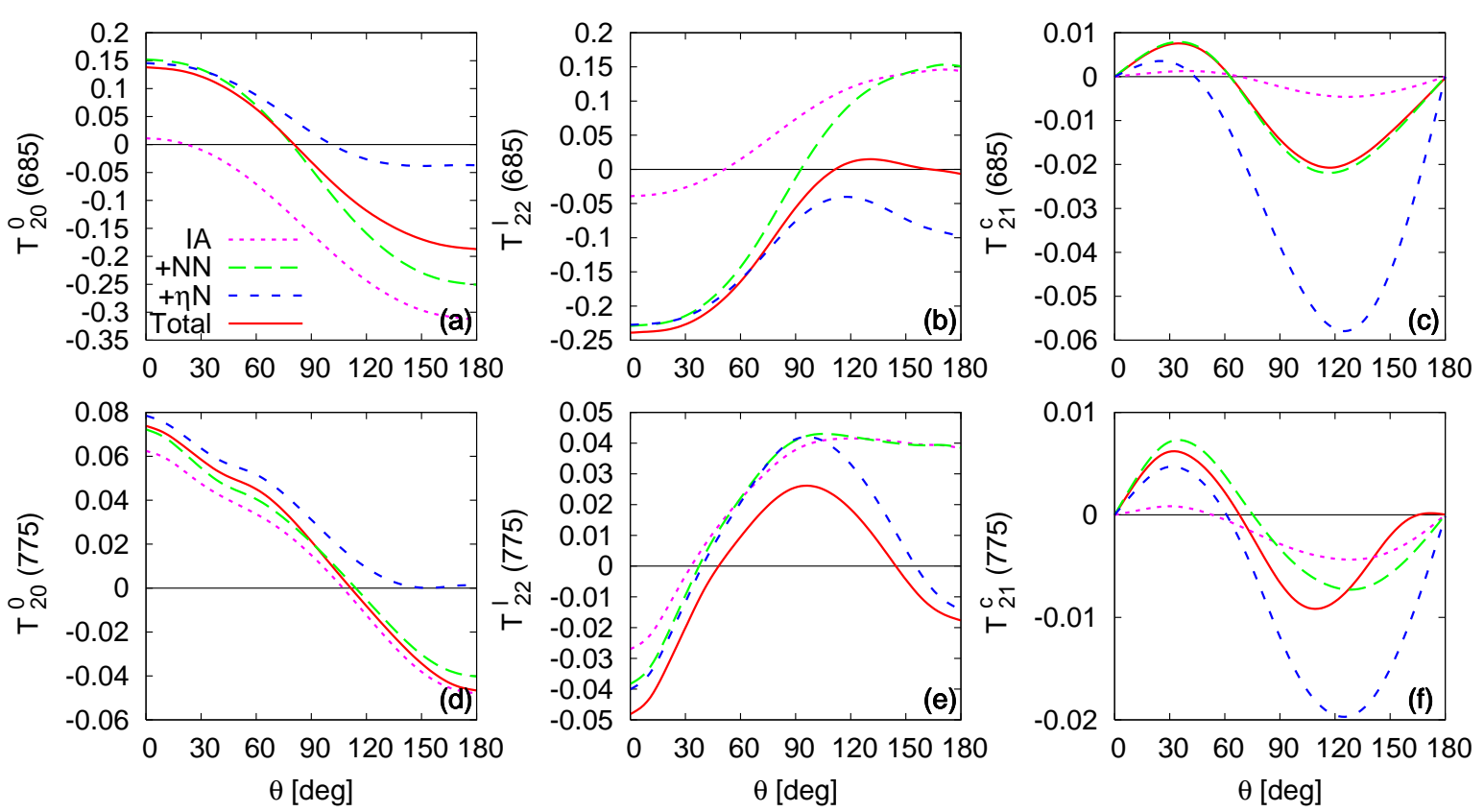

FIG. 7: (Color online) Polarization observables $T_{20}^{0}, T_{22}^{\ell}$ and $T_{21}^{c}$ of the semi-inclusive differential cross section for $\gamma d \rightarrow \eta n p$ at $E_{\gamma}=685$ and $775 \mathrm{MeV}$. Notation as in Fig. 3

3.825.2014/K). M. Tammam would like to thank the Egyptian Government for supporting him through the Post Doctor Program of Egypt. A. Fix and M. Tammam are also grateful for the kind hospitality of the Institut für Kernphysik of the Johannes Gutenberg-Universität Mainz.

[1] B. Krusche, J. Ahrens, J. R. M. Annand, G. Anton, R. Beck, M. Fuchs, A. R. Gabler and F. Haerter et al., Phys. Lett. B 358, 40 (1995).

[2] A. I. Fiks and V. A. Tryasuchev, Phys. Atom. Nucl. 60, 35 (1997) [Yad. Fiz. 60, 41 (1997)].

[3] C. Deutsch-Sauermann, B. Friman and W. Nörenberg, Phys. Lett. B 409, 51 (1997) nucl-th/9701022.

[4] A. Fix and H. Arenhövel, Z. Phys. A 359, 427 (1997) nucl-th/9706072.

[5] A. Fix and H. Arenhövel, Eur. Phys. J. A 9, 119 (2000) nucl-th/0006074.

[6] A. Fix and H. Arenhövel, Phys. Lett. B 492, 32 (2000) nucl-th/0008031.

[7] A. Sibirtsev, S. Schneider, C. Elster, J. Haidenbauer, S. Krewald and J. Speth, Phys. Rev. C 65, 067002 (2002) nucl-th/0203039.

[8] A. Fix and H. Arenhövel, Nucl. Phys. A 697, 277 (2002) nucl-th/0104032.

[9] A. Fix and H. Arenhövel, Eur. Phys. J. A 19, 275 (2004) nucl-th/0305098.

[10] A. Salam and H. Arenhövel, Phys. Rev. C 70, 044008 (2004) nucl-th/0407098.

[11] W.-T. Chiang, S.-N. Yang, L. Tiator, and D. Drechsel, Nucl. Phys. A 700, 429 (2002).

[12] D. Drechsel, S. S. Kamalov, and L. Tiator, Eur. Phys. J. A 34, 69 (2007).

[13] M. Batinić, I. Šlaus, A. Švarc, and B. M. K. Nefkens, Phys. Rev. C 51, 2310 (1995).

[14] C. Bennhold and H. Tanabe, Nucl. Phys. A 530, 625 (1991).

[15] A. M. Green and S. Wycech, Phys. Rev. C 55, 2167 (1997) nucl-th/9703009.

[16] R. A. Arndt, J. M. Ford, and L. D. Roper, Phys. Rev. D 32,1085 (1985).

[17] J. Haidenbauer and W. Plessas, Phys. Rev. C 30, 1822 (1984); Phys. Rev. C 32, 1424 (1985).

[18] R. Machleidt, Phys. Rev. C 63, 024001 (2001).

[19] V. G. J. Stoks, R. A. M. Klomp, C. P. F. Terheggen, and J. J. de Swart, Phys. Rev. C 49, 2950 (1994).

[20] H. Arenhövel and A. Fix, Phys. Rev. C 72, 064004 (2005).

[21] H. Arenhövel, A. Fix and M. Schwamb, Phys. Rev. Lett. 93, 202301 (2004). 